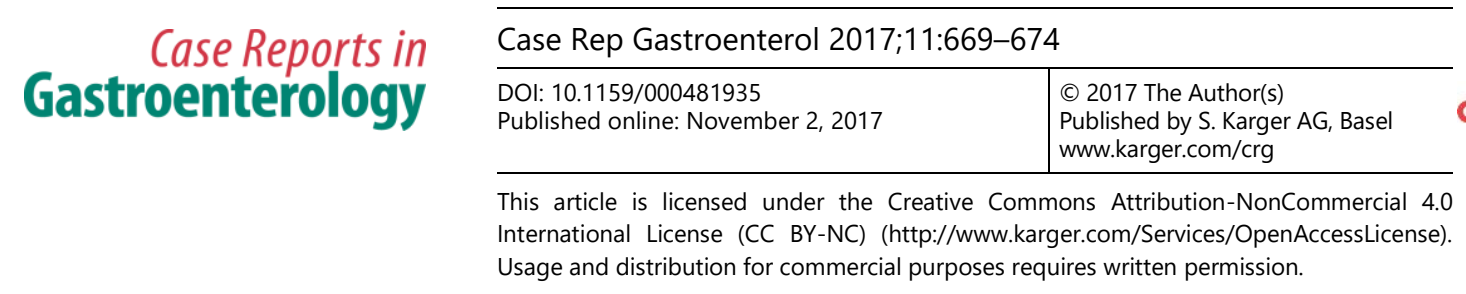

\title{
A Rare Case of Intraductal Tubulopapillary Neoplasm of the Pancreas Rupturing and Causing Acute Peritonitis
}

\author{
Akira Umemura $^{a} \quad$ Kazuyuki Ishida $^{\mathrm{b}}$ Hiroyuki Nitta $^{\mathrm{a}}$ Takeshi Takahara ${ }^{\mathrm{a}}$ \\ Yasushi Hasegawa $^{a}$ Kenji Makabe ${ }^{a} \quad$ Akira Sasaki $^{a}$ \\ aDepartment of Surgery, Iwate Medical University, Morioka, Japan; bDepartment of \\ Molecular Diagnostic Pathology, Iwate Medical University, Morioka, Japan
}

\section{Keywords}

Intraductal tubulopapillary neoplasm · Pancreatic intraductal neoplasm · Tubulopapillary growth $\cdot$ Rupture $\cdot$ Acute peritonitis · Peritonitis carcinomatosa

\begin{abstract}
An intraductal tubulopapillary neoplasm (ITPN) is a very rare pancreatic tumor. Here we report an extremely rare case of an ITPN rupturing and causing acute peritonitis. A 50-year-old woman presented with left flank pain and vomiting. A computed tomography (CT) scan revealed gigantic multilocular cysts in the pancreatic tail and massive fluid collection in the abdominal cavity. The serum, urine, and abdominal fluid amylase levels were highly elevated, so she was conservatively treated with intraperitoneal drainage and antibiotics for a diagnosis of ruptured pancreatic cysts. After this patient recovered, a CT scan revealed a 2-cm lowdensity mass located in the body of the pancreas. This was diagnosed as a pancreatic ductal adenocarcinoma of the pancreatic body with an intraductal papillary mucinous neoplasm, and a distal pancreatectomy was performed. The tumor was composed of cuboidal highgrade dysplastic cells proliferating in a tubulopapillary growth pattern without mucin production. An immunohistochemical examination revealed that the tumor cells were positive for MUC1 and CK7, but negative for MUC5AC. These features led to the final diagnosis of ITPN. In this case, the solid ITPN growth obstructed the lumen of the main pancreatic duct,
\end{abstract}


and the intraductal pressure of the distal side rose gradually. Then, pancreatic cysts formed and burst into the abdominal cavity when the intraductal pressure was at its maximum. However, an ITPN consists of high-grade atypical cells derived from the pancreatic ductal epithelium in principle, so the rupture may be an independent risk factor for peritonitis carcinomatosa in the future.

(C) 2017 The Author(s)

Published by S. Karger AG, Basel

\section{Introduction}

An intraductal tubulopapillary neoplasm (ITPN) of the pancreas is a very rare tumor that is characterized by intraductal tubulopapillary growth, ductal differentiation, scant intracellular mucin production, and cellular dysplasia [1]. ITPNs are estimated to account for less than $1 \%$ of all pancreatic exocrine tumors and $3 \%$ of all pancreatic intraductal neoplasms [2]. Because of the rarity of this disease, information regarding both the clinical and molecular aspects of an ITPN as a newly described entity is limited. It has recently become evident that ITPNs are less aggressive than other pancreatic malignancies, and that it is important to distinguish an ITPN from both an intraductal papillary mucinous neoplasm (IPMN) and pancreatic ductal adenocarcinoma (PDAC) [3].

Here we report an extremely rare case of an ITPN of the pancreas rupturing and causing acute peritonitis, with some considerations about the clinical mechanisms of ITPN ruptures.

\section{Case Presentation}

A 50-year-old woman presented with a 5-day history of left flank pain and vomiting. She came to our hospital with a complaint of worsening left flank pain and abdominal distension. Her severe symptoms and rebound tenderness evoked symptoms of an acute abdomen, and an abdominal computed tomography (CT) workup revealed gigantic multilocular cysts in the pancreatic tail and massive fluid collection in the abdominal cavity (Fig. 1a, b). Her laboratory data were almost within the normal range, except for the following: hemoglobin level was decreased $(9.8 \mathrm{~g} / \mathrm{dL})$, C-reactive protein level was slightly elevated $(3.3 \mathrm{mg} / \mathrm{dL})$, serum and urine amylase were elevated $(1,704$ and 11,386 U/L), and the other serum pancreatic enzymes including trypsin, phospholipase-A2, elastase-1, and lipase were highly elevated at $900 \mathrm{ng} / \mathrm{mL}, 5,210 \mathrm{ng} / \mathrm{dL}, 1,900 \mathrm{ng} / \mathrm{dL}$, and 2,460 U/L, respectively. Among the serum tumor markers, only the CA125 level was elevated $(89.8 \mathrm{U} / \mathrm{mL})$. To determine the origin of the acute abdomen, we performed intraperitoneal drainage and found the amylase level in the ascites to be highly elevated $(33,432 \mathrm{U} / \mathrm{L})$. Based on these findings, she was diagnosed as having acute peritonitis due to the rupture of the pancreatic cysts, and she was conservatively treated with intraperitoneal drainage and antibiotics.

After this patient's recovery from acute peritonitis, she received a contrast-enhanced CT to detect the cause of the pancreatic cyst ruptures. The contrast-enhanced CT revealed that a 2-cm low-density mass was located in the body of the pancreas, and that the peripheral side of the main pancreatic duct (MPD) was dilated (Fig. 1c). In addition, a magnetic resonance cholangiopancreatography also showed a dilatation of the MPD and gigantic pancreatic cysts (Fig. 1d). Although we performed an endoscopic retrograde cholangiopancreatography to confirm the origin of the dilatation of the MPD and formation of the gigantic pancreatic cysts, we could not cannulate into the MPD. Therefore, she was diagnosed as having a PDAC of the 
pancreatic body with an IPMN. We performed a distal pancreatectomy with a radical lymphadenectomy as a curative treatment for this pancreatic cancer.

The gross examination of the specimen showed no mucin and a solid tumor measuring $15 \times 10 \mathrm{~mm}$ in size invaginating into the MPD (Fig. 2). There was dilatation of the MPD and multiple cysts with severe inflammation located in the pancreatic tail (Fig. 2). Not only the MPD but also the branches of the pancreatic ducts were replete with tumor cells (Fig. 3a). The tumor was composed of cuboidal high-grade dysplastic cells proliferating in a tubulopapillary growth pattern (Fig. 3b); however, there was no mucin production (Fig. 3c), vascular invasion, perineural infiltration, or lymph node metastasis. An immunohistochemical examination was performed, as described elsewhere, indicating that the tumor cells were positive for MUC1 and CK7, but negative for MUC2, MUC5AC, and MUC6 (Fig. 3d-h). These features led to the final diagnosis of ITPN with noninvasive adenocarcinoma of the pancreas. The surgical margins were all negative. A molecular analysis was conducted to determine if there were any somatic mutations in the TP53, PIK3CA, KRAS, BRAF, and GNAS genes, but there were no mutations found. This patient was discharged from the hospital on postoperative day 12 without any complications, and no recurrence was noted for 2 years after the operation.

\section{Discussion}

An ITPN is an intraductal neoplasm of the pancreas first reported by Yamaguchi et al. [2] in 2009. According to the 2010 World Health Organization classification, the main characteristics of an ITPN are as follows: appearance of a solid nodular tumor obstructing dilated ducts upon macroscopic examination; no visible secreted mucin; tubulopapillary growth; uniform high-grade atypia throughout the neoplasm; easily recognizable necrotic foci; ductal differentiation, as indicated by MUC1 and CK7 expression; absence of acinar differentiation, as indicated by the absence of trypsin; absence of MUC2, MUC5AC, and fascine; and absence of KRAS and/or BRAF mutations [4]. The present case demonstrated all of the important histopathological characteristics of an ITPN. According to the molecular features of common pancreatic ductal neoplasms, IPMNs are frequently associated with mutations in KRAS and BRAF [4]; however, mutations in the genes involved in the PI3K pathway, including PIK3CA, PTEN, and AKT1, are very rare in common pancreatic ductal neoplasms $[5,6]$. The present case did not harbor any somatic mutations in TP53, PIK3CA, KRAS, BRAF, or GNAS. Some cases of ITPN harbor somatic mutations in PIK3CA and BRAF [4, 7]; therefore, further molecular analyses of more cases are warranted to clarify the impact of these mutations on the development and progression of ITPNs.

Our case is the first report of an ITPN with the formation of gigantic pancreatic cysts rupturing and causing acute peritonitis. Three cases of rupture have been reported so far in IPMNs $[8,9]$. Two of them caused pseudomyxoma peritonei due to the rupture of the pancreatic duct, in which the tumor cells spread through the fistula into the abdominal cavity. Imaoka et al. [10] previously reported a case of pseudomyxoma peritonei caused by acute pancreatitis in a patient with an IPMN, in which the mucus and pancreatitis may have raised the pressure in the pancreatic duct, leading to rupture and dissemination [8]. In our case, it is believed that the solid ITPN formation in the MPD obstructed the flow of pancreatic fluid and raised the pressure of the distal pancreatic duct. Gigantic pancreatic cysts then formed and ruptured when the pressure of the distal pancreatic duct was at its maximum. Overall, it seems that an ITPN is a relatively indolent neoplasm with a significantly better prognosis 
than a PDAC. However, we have to keep in mind the possibility of the onset of peritonitis carcinomatosa when the ITPN ruptures and tumor cells are disseminated in the abdominal cavity as in the rupture of an IPMN.

Little is currently known about the natural history of an ITPN, and this is mainly due to the rarity of the tumor. Only recently have its features been defined, with a lack of clear information in several publications on the presence and extent of the stromal invasion and confusion regarding the nomenclature [1]. Therefore, determining the best management strategy for an ITPN and improving the accuracy of the preoperative prognosis requires the collection and analysis of further epidemiological and pathological data.

\section{Conclusion}

We have reported the first case of an ITPN of the pancreas rupturing and causing acute peritonitis. In this case, a solid ITPN growth obstructed the lumen of the MPD, and the intraductal pressure of the distal side rose gradually. Pancreatic cysts then formed and burst into the abdominal cavity when the intraductal pressure was at its maximum. However, an ITPN consists of high-grade atypical cells derived from the pancreatic ductal epithelium in principle, so an ITPN rupture may cause peritonitis carcinomatosa in the future. For these reasons, in patients with a cystic lesion of the pancreas, an ITPN should be considered as part of the differential diagnosis.

\section{Statement of Ethics}

The authors have no ethical conflicts to disclosure. Informed consent was obtained from the presented patient.

\section{Disclosure Statement}

Akira Umemura and all the co-authors have no conflict of interest.

\section{References}

1 Date K, Okabayashi T, Shima Y, Iwata J, Sumiyoshi T, Kozuki A, Morita S, Hata Y, Noda Y, Nishioka A, Matsumoto M: Clinicopathological features and surgical outcomes of intraductal tubulopapillary neoplasm of the pancreas: a systematic review. Langenbecks Arch Surg 2016;401:1391-1396.

-2 Yamaguchi H, Shimizu M, Ban S, Koyama I, Hatori T, Fujita I, Yamamoto M, Kawamura S, Kobayashi M, Ishida K, Morikawa T, Motoi F, Unno M, Kanno A, Satoh K, Shimosegawa T, Orikasa H, Watanabe T, Nishimura K, Ebihara Y, Koike N, Furukawa T: Intraductal tubulopapillary neoplasms of the pancreas distinct from pancreatic intraepithelial neoplasia and intraductal papillary mucinous neoplasms. Am J Surg Pathol 2009;33:1164-1172.

-3 Yamaguchi H, Kuboki Y, Hatori T, Yamamoto M, Shimizu K, Shiratori K, Shibata N, Shimizu M, Furukawa $\mathrm{T}$ : The discrete nature and distinguishing molecular features of pancreatic intraductal tubulopapillary neoplasms and intraductal papillary mucinous neoplasms of the gastric type, pyloric gland variant. J Pathol 2013;231:335-341.

-4 Yamaguchi H, Kuboki Y, Hatori T, Yamamoto M, Shiratori K, Kawamura S, Kobayashi M, Shimizu M, Ban S, Koyama I, Higashi M, Shin N, Ishida K, Morikawa T, Motoi F, Unno M, Kanno A, Satoh K, Shimosegawa T, Orikasa H, Watanabe T, Nishimura K, Harada Y, Furukawa T: Somatic mutations in PIK3CA and activation of AKT in intraductal tubulopapillary neoplasms of the pancreas. Am J Pathol 2011;351:1812-1817. 


\section{Case Reports in Gastroenterology}

\begin{tabular}{l|l}
\hline Case Rep Gastroenterol 2017;11:669-674 \\
\hline DOI: 10.1159/000481935 & $\begin{array}{l}\text { ○ 2017 The Author(s). Published by S. Karger AG, Basel } \\
\text { www.karger.com/crg }\end{array}$ \\
\hline
\end{tabular}

Umemura et al.: A Rare Case of Intraductal Tubulopapillary Neoplasm of the Pancreas Rupturing and Causing Acute Peritonitis

5 Bleeker FE, Felicioni L, Buttitta F, Lamba S, Cardone L, Rodolfo M, Scarpa A, Leenstra S, Frattini M, Barbareschi M, Grammastro MD, Sciarrotta MG, Zanon C, Marchetti A, Bardelli A: AKT1(E17K) in human solid tumours. Oncogene 2008;27:5648-5650.

6 Samuels Y, Wang Z, Bardelli A, Silliman N, Ptak J, Szabo S, Yan H, Gazdar A, Powell SM, Riggins GJ, Willson JK, Markowitz S, Kinzler KW, Vogelstein B, Velculescu VE: High frequency of mutations of the PIK3CA gene in human cancers. Science 2004;304:554.

-7 Urata T, Naito Y, Nagamine M, Izumi Y, Tonaki G, Iwasaki H, Sasaki A, Yamasaki A, Minami N, Yoshioka R, Kitada H, Takekuma Y, Yokomizo H, Fukuda S, Yamaguchi H, Kuboki Y, Furukawa T, Hifumi M: Intraductal tubulopapillary neoplasm of the pancreas with somatic BRAF mutation. Clin J Gastroenterol 2012;5:413-420.

-8 Imaoka H, Yamao K, Hijioka S, Hara K, Mizuno N, Tanaka T, Kondo S, Tajika M, Shimizu Y, Niwa Y: Pseudomyxoma peritonei arising from intraductal papillary neoplasm after surgical pancreatectomy: report of 2 cases and review of the literature. Clin J Gastroenterol 2012;5:15-19.

-9 Shimizu Y, Imaizumi H, Yamauchi H, Okuwaki K, Miyazawa S, Iwai T, Takezawa M, Kida M, Suzuki E, Saegusa M, Koizumi W: Pancreatic fistula extending into the thigh caused by the rupture of an intraductal papillary mucinous adenoma of the pancreas. Intern Med 2017;56:307-313.

-10 Imaoka H, Yamao K, Salem AA, Mizuno N, Takahashi K, Sawaki A, Isaka T, Okamoto Y, Yanagisawa A, Shimizu Y: Pseudomyxoma peritonei caused by acute pancreatitis in intraductal papillary mucinous carcinoma of the pancreas. Pancreas 2006;32:223-224.
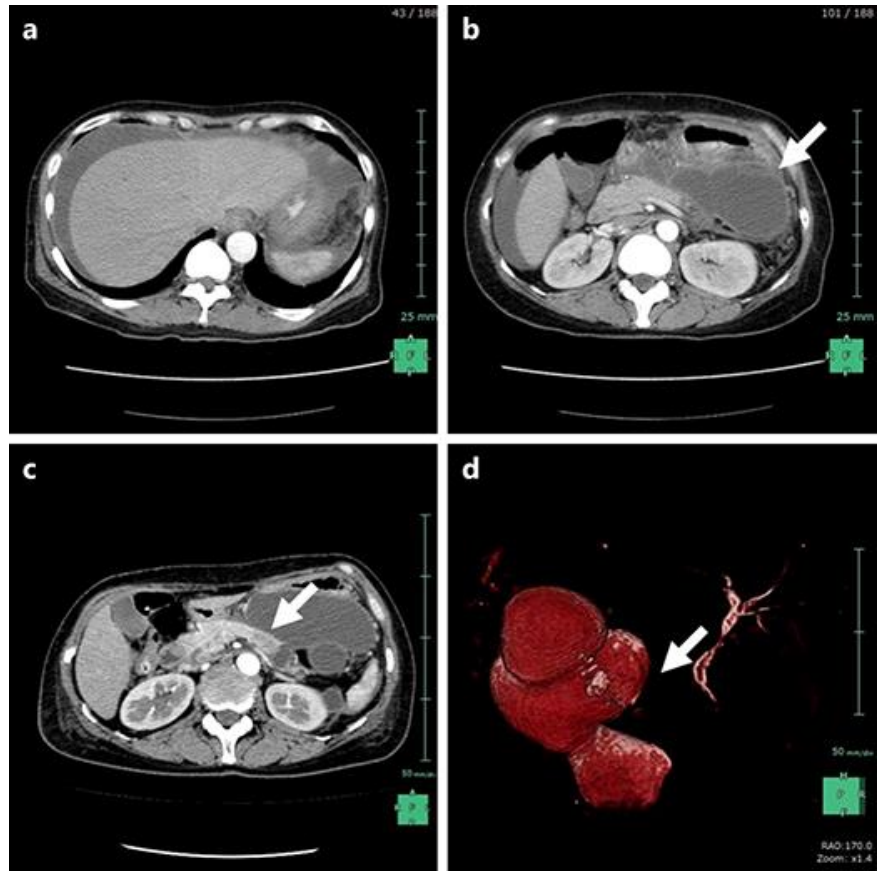

Fig. 1. a, b A contrast-enhanced computed tomography (CT) of the abdomen on admission day revealed massive fluid collection in the abdominal cavity and gigantic multilocular cysts of the pancreatic tail (white arrow). c A contrast-enhanced CT after recovery from acute peritonitis revealed the 2 -cm low-density mass located at the pancreas body (white arrow) and the peripheral main pancreatic duct (MPD) dilatation. d A magnetic resonance cholangiopancreatography also showed interruption of the MPD (white arrow) and dilatation of the peripheral MPD dilatation. 


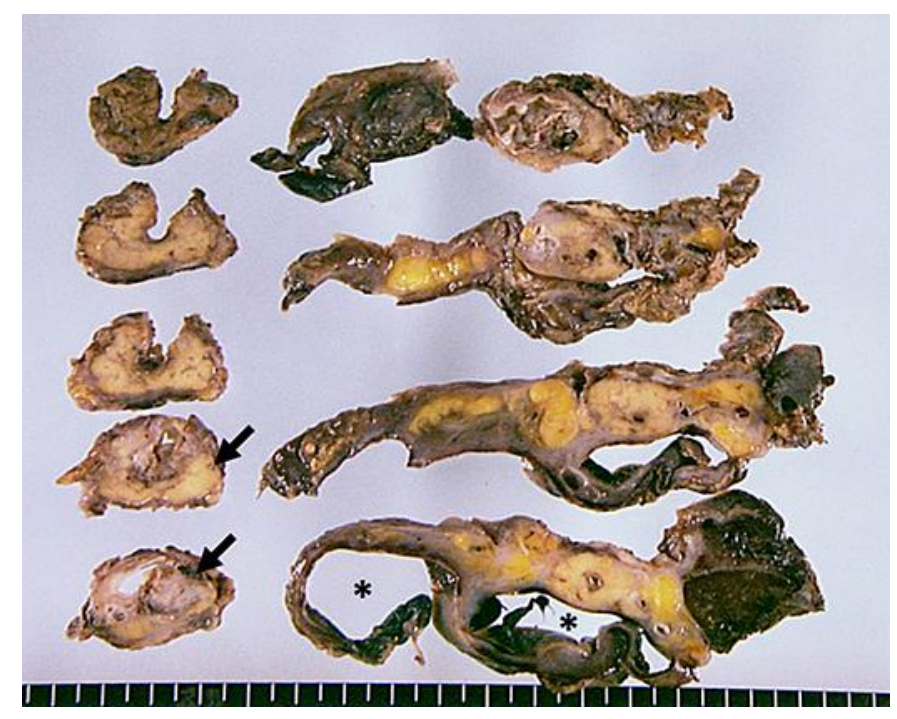

Fig. 2. The gross examination of the specimen showed no mucin and a solid tumor measuring $15 \times 10 \mathrm{~mm}$ in size invaginating into the main pancreatic duct (MPD) (black arrows). There was dilatation of the MPD and multiple cysts with severe inflammation located in the pancreatic tail (black asterisks).

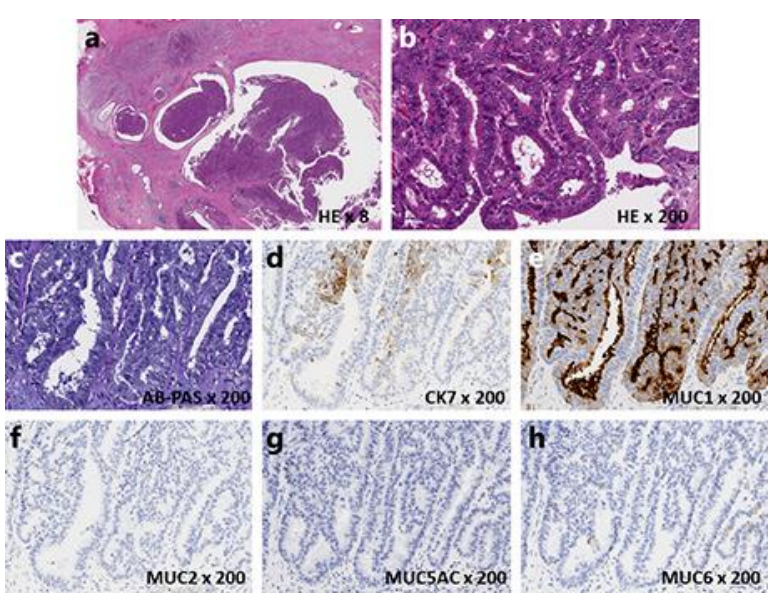

Fig. 3. a A low-magnification image showing an intraductal tubulopapillary neoplasm. Not only the main pancreatic duct but also the branches of the pancreatic ducts were replete with tumor cells. b A highmagnification image revealed that the tumor was composed of solid proliferating tumor cells with tubular or papillary formation with high-grade dysplasia. c There was no mucin that was detectable by AB-PAS stain. $\mathbf{d}-\mathbf{h}$ An immunohistochemical examination revealed that the tumor cells were positive for CK7 and MUC1, but negative for MUC2, MUC5AC, and MUC6. 